\title{
Advances in Spatial Risk Analysis
}

Nikolaos Argyris ${ }^{a}$, Valentina Ferretti ${ }^{\mathrm{b}, \mathrm{c}}$, Simon French ${ }^{\mathrm{d}}$, Seth Guikema ${ }^{\mathrm{e}}$, Gilberto Montibeller ${ }^{\mathrm{a}}$,

${ }^{a}$ School of Business and Economics, Loughborough University

${ }^{b}$ Politecnico di Mílano

${ }^{c}$ London School of Economics and Political Science

${ }^{d}$ University of Warwick

${ }^{\mathrm{e}}$ University of Michigan

\section{1}

口

1

Introduction

We live in a 3D world with increasing availability of real time spatial data, from satellite information, mobile tracking, autonomous transportation systems, among many other sources. Such availability of data, together with increasingly sophisticated risk and decision analytic frameworks, is enabling more effective support in answering questions such as:

- How to plan the growth of cities in a sustainable way?

- Where to bury nuclear waste?

- Where to increase flood defenses?

- What is the likely spatial spread of a disease?

- How will airborne or waterborne contamination disperse?

- What is the likely impact region of a satellite in a decaying orbit?

- How to distribute police on open patrol in a large city?

- How to assess the chance of finding a missing aircraft over a wide area?

In answering these questions, there is growing recognition that the spatial distribution of many of the factors affecting the risks, costs and outcomes are heterogeneous (e.g. Keller et al. 2014, Zagmutt et al., 2015, Zhou et al., 2014). The rich literature on multi-criteria spatial decision support systems (Malczewski, 2006) has also seen an increasing number of applications involving risk assessment (e.g. Aceves-Quesada et al., 2007; Vadrevu et al., 2010). In parallel with the analysis of spatial risk, the presentation of spatial and geographical uncertainty has also attracted growing attention (MacEachren et al. 2005).

The time is thus ripe for having a special issue on Spatial Risk Analysis, which we have edited. The aims of the special issue were to:

(i) gather recent theoretical and applied developments in the field;

(ii) identify common trends and new directions of research;

(iii) provide some coherence to this active field of research.

We are delighted that the response to our call for papers has been so positive, with 45 submissions, the highest number of submissions for a special issue in Risk Analysis history. This success confirms the widespread and fast-growing interest on the topic. From the initial set, 17 papers have been accepted after the reviewing process. This collection of papers includes both theoretical and applied studies, which deal with different types of risks (e.g. environmental, industrial, health) and cover different stages of risk analysis processes.

This is the author manuscript accepted for publication and has undergone full peer review but has not been through the copyediting, typesetting, pagination and proofreading process, which may lead to differences between this version and the Version of Record. Please cite this article as doi:

$\underline{10.1111 / \text { risa. } 13260 .}$.

This article is protected by copyright. All rights reserved. 
Applications cover risks related to business, biosecurity, biological invasion, civil aviation, climate change vulnerability, contamination, groundwater induced subsidence, emergency risk analysis, flooding and extreme rain events, power networks, radiation dose assessment, railways, subsidence, and tornados.

In the next section, we suggest a five-stage framework which may help to position this special issue within the fast-growing field of research outlined above. Subsequently, we identify key complexities in each stage of the framework brought by the spatial dimension of the assessment. We then discuss the contributions of the papers in this special issue for each stage in the proposed framework. We conclude with some directions for further research in this emerging field, by identifying some potential gaps as well as strengths for the consolidation of the topic.

\section{$2 \quad$ Key Steps and Complexities in Spatial Risk Analysis}

Based on our experiences in conducting risk analysis (Francis et al. 2011; French, 1996; Han et al. 2009, French et al, 2016; Guikema 2009; Guikema et al. 2014; Papamichail and French, 2013) as well as spatial decision analysis (Ferretti and Montibeller, 2016) we propose the following conceptualization of spatial risk analysis processes (Figure 1). For each stage, represented by a box, we identify the corresponding inputs and outputs, with the latter becoming an input to the next stage. We emphasise that the analytic process is interactive, constructive and, above all, provides a learning experience for the risk-owners and stakeholders.

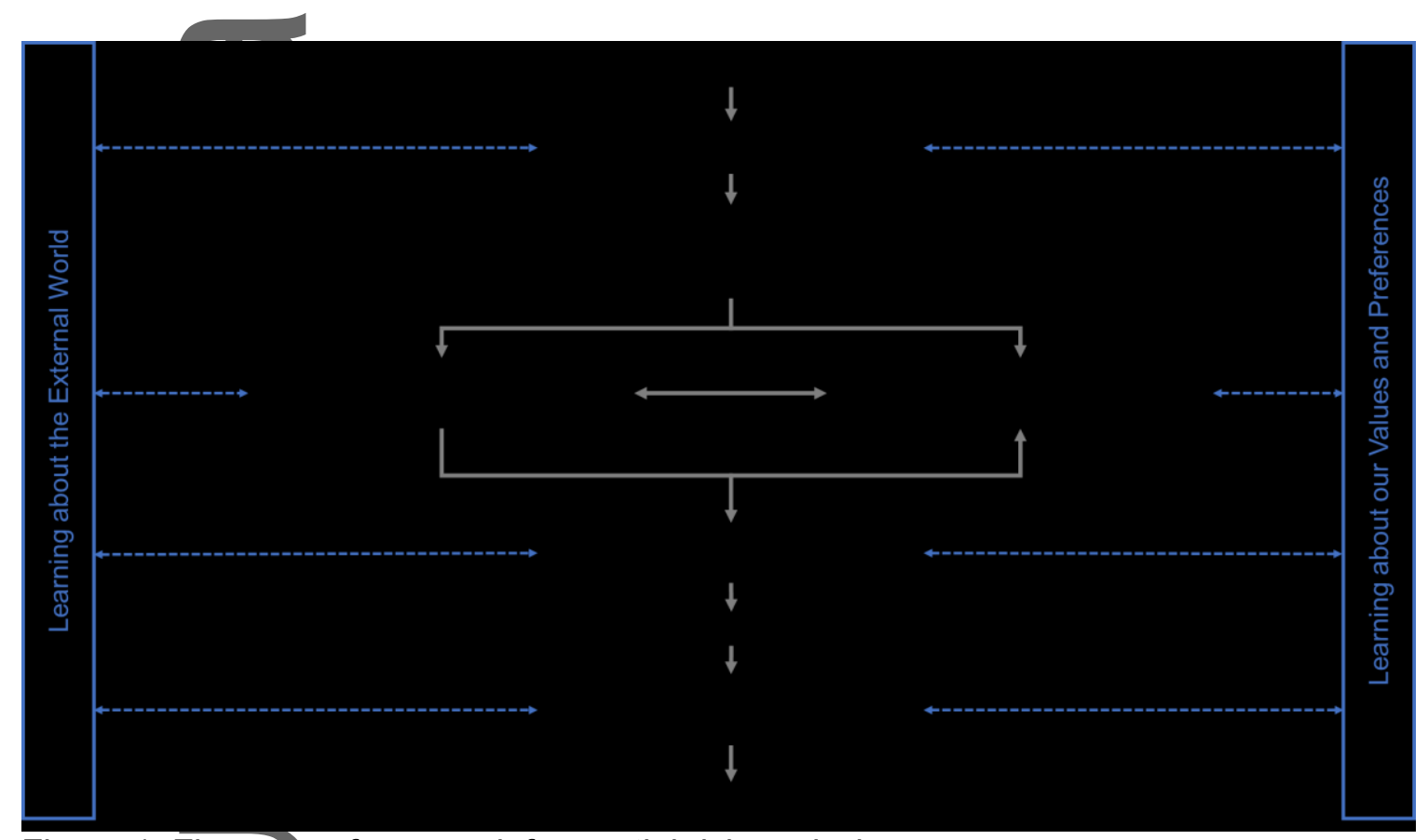

Figure 1. Five-stage framework for spatial risk analysis.

The first stage of any risk analysis consists in structuring the context of the intervention and providing scope to the analysis. Key activities in this stage consist in identifying stakeholders, understanding the main uncertainties, locating spatial alternatives, and agreeing on objectives for project appraisal. Once these characteristics are identified, the analyst will work with the risk-owner and stakeholders to select an adequate assessment method as well as specify component variables and target variables.

In stage two, i.e. predictive modelling and forecasting, spatial distributions of component variables will be defined, and their overall impact assessed. When evidence is missing and 
simulation models cannot be developed, expert judgment may provide the necessary estimates for the analysis (Dias et al, 2018). In parallel, preferences and values relating to potential impacts need to be elicited and explored, often for several stakeholders' groupings. Although Stages 2 and 3 may be separated conceptually, in practice they will interact. Predictive modelling will identify that impacts need to be evaluated with preference and value modelling and, in turn, preference and value modelling may identify further aspects of the impacts that need predicting.

The input to the fourth stage, i.e. robustness analyses, is a spatial distribution of outcomes across the area under analysis. This stage tests the stability of the analytical results against variations in model parameters. Its result is an evidence-based recommendation for resource allocation and/or mitigation measures. A successful intervention, after the final stage of communication and presentation of results, will have as output an action plan agreed among the key stakeholders, as well as the decision makers' commitment to its implementation

In the following sections, we attempt to identify the main complexities associated to each of the above stages of spatial risk analysis. We also cluster the special issue's papers based on the stage that they deal with and summarise their key contribution.

\subsection{Stage 1: Problem Structuring}

Problem structuring is a crucial first step of any risk and decision analysis process, given the importance of agreeing on who should be involved in the analysis, on the problem framing, on the key components of the evaluation (uncertainties, objectives, alternatives), which jointly will lead to the selection of a suitable assessment method. There is extensive literature on supporting this stage with useful guidelines on how to use these qualitative problem structuring methods, e.g. causal maps, causal loop diagrams, and soft systems methodology (Rosenhead and Mingers, 2001; Shaw et al., 2006). However, most of this literature addresses problems from a non-spatial perspective.

If we are instead engaged in a spatial risk analysis, the following complexities may make this stage more challenging. First, the geographical distribution of stakeholders may affect the evaluation of impacts and may require geographical procedural justice to ensure fair representativeness across the area. Second, existing problem structuring methods are currently not designed to visualise geographical factors within their qualitative models. Third, conceptualising geographical uncertainty is surprisingly difficult since natural language may merge several distinct concepts.

Gardezi and Arbuckle's paper in this special issue addresses a preliminary stage of spatial risk analysis by exploring whether specific model parameters, in this case perceived farmers' capacity against extreme rain events, should be considered in the modelling stage. They develop a vulnerability index that incorporates both objective and perceived attributes of adaptive capacity and suggest that vulnerability assessments relying only on objective measures might miss important socio-cognitive dimensions of capacity.

\subsection{Stage 2: Predictive Modelling and Forecasting}

Predictive modelling and forecasting provide estimates of the probability distribution of outcomes or events and thus represent a core activity in any risk analysis (Bedford and Cooke, 2001). Typically, predictive models require historical data and/or simulations to infer the impacts of events. Within this context, expert judgment is often used whenever specific data is sparse or lacking (Dias et al., 2017). 
In spatial risk analysis, this stage becomes more challenging due to the presence of spatially distributed parameters, complex geographical co-variation, and availability of big data for spatio-temporal predictive models. In addition, the number of judgments required from experts can become unmanageable if experts have to be consulted when data is sparse and spatially spread out. The majority of papers in this special issue focused on this stage and are briefly described next.

Calabrese et al. propose a method for detecting the risk of small and medium enterprises defaults by considering the enterprises' location and their demographic characteristics. They found that taking into account this information helped to improve the ability to predict defaults of non-start-ups in London.

Cope et al. study the risk of biological invasions associated with particular transport pathways and source regions by adapting the "range bagging" method to determine environmental/matching between areas. They classify patterns of global invasion into Australian states and territories, validate it and produce a list of high-risk species not previously known to be present in Australia. They find that geographic distance is an important predictor of the potential risk of invasive species, in addition to transport and environmental similarity.

Sundell et al. present a novel method for combining a probabilistic soil stratification model with statistical analysis of compression parameters for simulation of subsidence on a large area with a simple nonlinear 1-dimensional compression model. The results of this simulation include spatially explicit probabilistic estimates of subsidence magnitude and is used to create risk maps where areas with significant risk for subsidence are distinguished from low-risk areas.

Walker et al. develop a spatio-temporal exposure-hazard model, in which a dynamic model is built for quantifying concentration of contaminants in space and time. The model is tested on a simulation study where the impact of genetically modified maize is assessed on nontargeted insects.

The papers of Hu et al, Avelino and Dall'ebra, Ouyang and Trakas examine spatial impact assessment in interconnected systems. Hu et al consider the effects of hazards in network systems, examining how local hazards can spread out through the network topology. Two vulnerability indices are proposed for such systems, taking into account hazard location, affected area and direct and indirect hazard impacts. The paper also examines alternative ways of assessing impact by concentrating on different aspects of the network system topology.

Avelino and Dall'erba consider the case of economic impacts of natural disasters taking into account ripple effects due to business interruption. The paper examines the use of five different models in the literature for assessing economic losses in interconnected systems and offers a review of how future events can be assessed, as well as guidance on model selection.

In a similar vein, Ouyang et al examine failures in networks that form critical infrastructures, specific the Chinese Railway System. Discussing the concept of a spatially localised failure (SLF), they consider infrastructure vulnerability failures at a given node. They consider how to identify nodes whose failure would cause major impacts because of their connectivity and how these risks can be quantified. Drawing on examples from the Chinese railway networks for illustration, they demonstrate their approach. 
The paper of Trakas presents an approach for online risk analysis for evaluating the spatiotemporal impacts of extreme events on power networks and assessing system resilience. To capture the spatial risk on the system the paper introduces a severity risk index to capture probability and impacts on different network branches during an extreme event. Computing the risk index online can allow for real-time mitigation decisions during extreme events.

The paper of Robertson considers the case of spatial transmission models. Such models can be used to examine how phenomena like diseases, fires, rumours, among others, spreadacross a spatial system. The paper provides a review and a taxonomy of a wide variety of available modelling techniques, as well as recommendations to guide the choice of an appropriate model for a given context.

Schneeberger et al provide a proof-of-concept study on the use of a probabilistic framework for risk analysis of widespread flood events. The study introduces a flood-risk modelling framework consisting of three components: a hazard module for the expected water levels for all points in considered area; an impact module is used to characterize potential adverse consequences of flooding; and a risk assessment module combines the results to calculate expected and maximum values of flood impact indicators.

Terti et al consider the specific case of flash floods, recognising that the impacts of these depend much more on the dynamics of the local social-economic patterns. Traffic flows, work patterns, building occupancies, among other factor, vary with the time of day and days of the week. Thus, vulnerabilities are harder to establish. Using data from Texas and Oklahoma, they examine the risk of fatality in vehicle-related incidents during flash floods.

The ongoing risk of future nuclear accidents are a major concern to much of the public. The dose received by any population group predicts their risk of cancer. In their empirical study, Takahara et al. compare estimates of the dose actually received by four population groups after the Daiichi Fukushima Disaster with the predictions made at the time of the accident.

\subsection{Stage 3: Preference Modelling and Value Aggregation}

Whenever-we are confronted with multiple impacts or non-linear preferences over prospects, there is a peed to elicit value trade-offs among conflicting objectives as well as the risk attitudes of risk owners and stakeholders. Well-established protocols have been extensively used for non-spatial decisions (von Winterfeldt and Edwards, 1986; Dias et al., 2017), helping decision makers and stakeholders to represent their preferences and priorities in complex assessments. If preference modelling is needed in a spatial risk analysis, three extra complexities should be highlighted. First, traditional preference elicitation protocols will become more cognitively demanding as they have to be expressed over spatially spread out data and taking into account spatial relations (e.g. continuity of cells with similar values, proximity to sensitive areas, etc.). Second, the comparison of spatial alternatives typically requires an overall assessment of maps (with an aggregation of spatially distributed values into a single value index) which is complex due to such spatial relations (Metchebon et al., 2013). Third, potential variation among the effects of different hazards across regions, areas or groups of people may bring out concerns about equitability and fairness in remediation strategies, which need to be appropriately captured in preference modelling and decision making (see e.g. Karsu, Morton and Argyris, 2018).

The paper by Keller and Simon in this special issue considers the problem of the numerical representation preferences over spatially-varying outcomes by cardinal preference functions. The paper offers conditions for the existence of such preference functions for the cases where outcomes are described by a single attribute or multiple attributes. Assessment of such functions allows for evaluating decision problems where alternative outcomes need to be compared, or a choice among them be made. 
The paper by Ferretti and Montibeller in this special issue proposes an integrated framework for spatial risk analysis when the considered impacts are multi-dimensional in nature. The proposed framework decomposes the problem into the assessment of three spatially-varying components: probabilities of adverse outcomes, vulnerabilities and multi-dimensional impacts, which are aggregated through the use of multi-attribute preference functions.

\subsection{Stage 4: Robustness Analyses}

Robustness analyses help stakeholders and decision makers to better understand the consequences of setting up different priorities, varying the shape of utility functions, and considering the uncertainties in the data and in the model parameters. This type of analysis also improves communication of results and helps in identifying if more data on certain aspects need to be collected. However, they become more challenging in the spatial context (Ferretti and Montibeller, 2016) for three reasons. First, the data spread may impose a high computational load on the model. Second, it is unclear how to judge geographical sensitivity of the results and decide when a result should be considered sensitive given the changes in values in the final maps. Third, there might be cognitive and conceptual difficulties in dealing with geographically varying risks (e.g. in map-comparison tasks).

While the robustness of different systems exposed to a variety of hazards is the focus of several papers in this Special Issue, only few papers have considered the robustness of the results with respect to assumptions and model parameters. In their case-study of the Chinese Civil-Aviation Network, Hu et al. consider three scenarios for the spatial distribution of local hazards. Sundell et al. consider three scenarios for water-drawdown measurements in their study of potential subsidence induced by the excavation of a planned power-line tunnel in Stockholm. Ferretti and Montibeller consider the sensitivity of their proposed model for multi-impact spatial risk analysis to changes in the criterion weights of an additive utility function used to aggregate different impact categories. Finally, a number of papers in this Special Issue address robustness by using a probabilistic approach in their Predictive Models and Forecasts. Schneeberger et al. and Terti et al. develop probabilistic models for flood events. Takahara et al develop a probabilistic model for radiation dose assessment.

\subsection{Stage 5: Communication and Presentation of results}

Typically, the results of a risk or decision analysis are communicated via a static presentation of a small number of selected scenarios obtained through a back-room analysis. An interactive analysis with the decision makers, with real-time visualization of changes in the maps (e.g. using touch screen tables and online tools) may help stakeholders and decision makers to better develop a sense of ownership of the final recommendation as well as agree on an action plan (Ferretti and Montibeller, 2016).

However, communicating uncertainty in a spatial context is a difficult task (MacEachren et al. 2005) as the visualization of uncertainty may lead to misinterpretation and behavioral issues. For instance, contours of an effect can be confused with probability contours; or a lack of standardisation in symbols can mean maps become very difficult to decipher without constant reference to a legend. Finally, it might be challenging to communicate the results to spatially-distributed and culturally diverse stakeholders.

In this special issue, French et al. review the literature associated with spatio-temporal uncertainties in emergency risk analysis from both technical and behavioural perspectives. They suggest the use of multiple scenarios for exploring the consequences of spatiotemporal uncertainties and communicating them to decision makers. 
Meteorological offices regularly face the problem of advising the authorities and warning the public of severe weather events. How can this be done most effectively, if there is to be a balanced response without risking panic? Jon et al. in this special issue consider the case of tornados in the USA. They note that little research has been conducted into how people actually interpret and respond to the warning polygons used by the national weather service. Their research has many interesting findings, including the fact that the public are optimistic about their personal safety, but more pessimistic about the safety of their possessions such as cars, buildings, etc.

The previously mentioned paper by Cope et al. contribute to this stage by developing an interactive web-app so users may observe how risk of invasive species changes with changes in the model parameters. Sundell et al., also mentioned before, show how mapped risk areas and the results of the sensitivity analysis could be used to support decision making and communication with stakeholders about mitigation and monitoring measures. They mention the case for the planned City Link tunnel in Stockholm, where their maps have been successfully used for risk communication in legal court in the application for permit to modify groundwater conditions.

\section{Directions for Further Research}

Table 1 summarises what we judge as the key complexities for the five stages that we proposed for spatial risk analysis interventions.

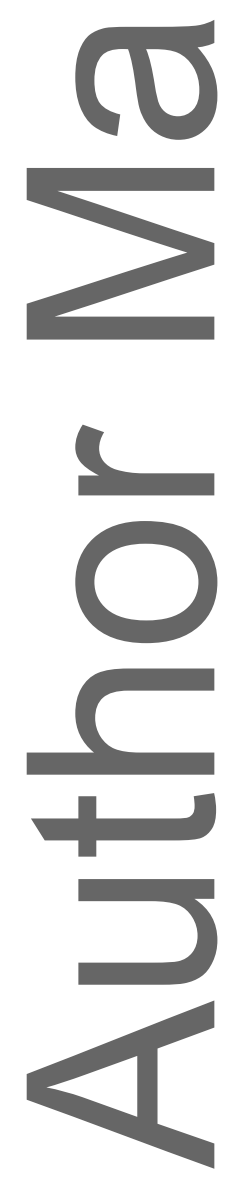


Table 1 Stages and associated complexities in spatial risk analysis

\begin{tabular}{|c|c|}
\hline Stages & Complexities \\
\hline Problem Structuring & $\begin{array}{l}\text { - The geographical distribution of stakeholders may } \\
\text { affect the evaluation of impacts and may require } \\
\text { geographical procedural justice. } \\
\text { - Existing problem structuring methods are currently } \\
\text { not designed to visualise geographical factors within } \\
\text { their qualitative models. }\end{array}$ \\
\hline $\begin{array}{l}\text { Predictive Modelling and } \\
\text { Forecasting }\end{array}$ & $\begin{array}{l}\text { - Presence of many parameters, complex } \\
\text { geographical co-variation, and availability of big } \\
\text { data for spatio-temporal predictive models. } \\
\text { - Expert judgement elicitation load can become } \\
\text { unmanageable as data become sparse. }\end{array}$ \\
\hline $\begin{array}{l}\text { Preferenc } \\
\text { Value Agg }\end{array}$ & $\begin{array}{l}\text { - More cognitively demanding preference elicitation } \\
\text { protocols resulting from relating preferences to } \\
\text { geographic factors and regions. } \\
\text { - Aggregation of spatially distributed values into a } \\
\text { single value index for map comparison. } \\
\text { - Agreeing equitable distribution of costs and benefits } \\
\text { across regions. }\end{array}$ \\
\hline Robustn & $\begin{array}{l}\text { - Very high computational load. } \\
\text { - Unclear how to judge geographical sensitivity of the } \\
\text { results across the several outputs of the SA (when } \\
\text { is a result considered sensitive given the changes in } \\
\text { values in the map?). } \\
\text { - Cognitive and conceptual difficulties of dealing with } \\
\text { geographically varying risk (e.g. in map-comparison } \\
\text { tasks). }\end{array}$ \\
\hline $\begin{array}{l}\text { Communication and } \\
\text { Presentation of results }\end{array}$ & $\begin{array}{l}\text { - } \text { Difficulty in visualising geographical uncertainty. } \\
\text { - Behavioural issues associated to uncertainty } \\
\text { communication. } \\
\text { - Communicating to spatially distributed and culturally } \\
\text { diverse stakeholders. }\end{array}$ \\
\hline
\end{tabular}

As we noted, the majority of the papers in this issue relate to Stage 2 in the framework (Figure 1); there seems to be relatively less research in the other stages. This suggests that the risk analysis community may need to consider whether spatial risks bring complexities into Stages 1, 3, 4 and 5 that need further processes, modelling tools and interventions to be developed and introduced to our tool kits. We believe that they do.

Moreover, we are aware that in focusing on distinct stages, cross-cutting issues may lose emphasis but many of them are highly relevant. For instance, behavioural heuristics and biases arise in all aspects of risk management (Aven and Zio, 2014; Montibeller and von Winterfeldt, 2015). The perception of and response to spatial risks are poorly researched in contrast with nonspatial ones (Slovic, 2000). Spatial risks often also have a temporal component and that can add to the difficulty in conceptualising, analysing and communicating aspects of the risk to decision makers. There is a need for many more studies to understand the cognitive issues associated with uncertainty visualisation and communication in all five stages. We note too that expert judgement is an important input to many analyses when specific data are sparse or lacking; however, structured expert judgement methodologies (Dias et al., 2018) hardly consider spatial uncertainties. 
Therefore, we hope that this special issue helps to further promote innovative applications of spatial risk analysis as well as inspire further theoretical developments in this relevant and growing field for risk analysis.

\section{Reference}

Aceves-Quesada J.F., Díaz-Salgado J., López-Blanco J. (2007). Vulnerability assessment in a volcanic risk evaluation in Central Mexico through a multi-criteria-GIS approach. Natural Hazards, 40, 2, 339-356.

Avelino A.F.T., Dall'erba S. (2018). Comparing the economic impact of natural disasters generated by different input-output models. An application to the 2007 Chehalis River Flood (WA). Risk Analysis , 39(1),

Aven T. and Zio E. (2014). Foundation issues in risk assessment and risk management. Risk Analysis, 34, 7, 1164-1172.

Bedford, T. \& R. Cooke (2001). Probabilistic Risk Analysis: Foundations and Methods. Cambridge, Cambridge University Press.

Calabrese R., Andreeva G., Ansell J. (2018). "Birds of a Feather" Fail Together: Exploring the Nature of Dependency in SME Defaults. Risk Analysis , 39(1),

Cope R.C. Ross J.V., Wittmann T.A., Watts M.J., Cassey P. (2018). Predicting the Risk of Biological Invasions Using Environmental Similarity and Transport Network Connectedness. Risk Analysis , 39(1),

Dias L.C., Morton A., Quigley J. (2018). Elicitation. The science and art of structuring judgments. Springer: Cham (Switzerland).

Ferretti V, Montibeller G. (2016). Key challenges and meta-choices in designing and applying multi-criteria spatial decision support systems. Decision Support Systems, 84, 4152.

Ferretti V. Montibeller, G. (2018). An iterated Framework for Environmental Multi-Impact Spatial Risk Analysis. Risk Analysis , 39(1),

Francis, R. A., S. M. Falconi, R. Nateghi, and S. D. Guikema. (2011). Probabilistic life cycle analysis model for evaluating electric power infrastructure risk mitigation investments. Climatic change, $106,31-55$

French, S. (1996). Multi-attribute decision support in the event of a nuclear accident. Journal of Multi-Criteria Decision Analysis, 5, 39-57.

French, S., Argyris, Haywood, S, N, Hort, M, Layton, H, Smith, J.Q., Presenting Uncertain Information in Radiological Emergencies. (2016) UK Atmospheric Dispersion Modelling Liaison Committee. Available at: https://admlc.wordpress.com/publications/.

French S., Argyris N., Haywood S.M., Hort M.C., Smith J.Q. (2018). Communicating Geographical Risks in Crisis Management: The Need for Research. Risk Analysis , 39(1),

Gardezi M., Arbuckle Jr J.G. (2018). Spatially representing vulnerability to extreme rain events using Midwestern farmers' objective and perceived attributes of adaptive capacity. Risk Analysis , 39(1), 
Guikema, S. D. (2009). Natural disaster risk analysis for critical infrastructure systems: An approach based on statistical learning theory. Reliability Engineering \& System Safety,94,4, 855-860.

Guikema, S. D., R. Nateghi, S. M. Quiring, A. Staid, A. C. Reilly \& M Gao. (2014). Predicting Hurricane Power Outages to Support Storm Response Planning. IEEE Access, 2, 1364-1373.

Han, S.R., Guikema, S.D., Quiring, S.M., Lee, K.H., Rosowsky, D. and Davidson, R.A., (2009). Estimating the spatial distribution of power outages during hurricanes in the Gulf coast region. Reliability Engineering \& System Safety, 94, 2,199-210.

Hu X., Li H., Guo X., van Gelder P.H.A.J.M., Shi P. (2018). Spatial Vulerability of Network Systems under Spatially Local Hazards. Risk Analysis (in press).

Kahneman, D. (2011). Thinking, Fast and Slow. London, Penguin, Allen Lane.

Karsu Ö., Morton A., Argyris N. (2018).. Capturing preferences for inequality aversion in decision support. European Journal of Operational Research,264, 2, 686-706.

Keller L.R., Kirkwood C.W., Simon J. (2015). Decision Analysis with Geographically Varying Outcomes: Preference Models and Illustrative Applications. Operations Research, 62, 1, 182-194.

Keller R., Simon J. (2 018).Preference Functions for Spatial Risk Analysis. Risk Analysis , 39(1),

Jon, I., Huang, S., Lindell, M. (2018). Perceptions and Expected Immediate Reactions to Severe Storm Displays. Risk Analysis , 39(1),

MacEachren A.M.,Robinson, A., Hopper, S., Gardner, S., Murray, R., Gahegan, M., \& Hetzler, E. (2005). Visualizing geospatial information uncertainty: what we know and what we need to know. Cartography and Geographic Information Science, 32, 3, 139-160.

Malczewski J. (2006). GIS-based multicriteria decision analysis: a survey of the literature. International Journal of Geographical Information Science, 20, 7, 703-726.

Metchebon T. SA, Brison V, Pirlot M. (2013).Two models for comparing decisional maps. International Journal of Multicriteria Decision Making, 3, 2-3, 129-156.

Montibeller G. \& von Winterfeldt D. (2015). Cognitive and motivational biases in decision and risk analysis. Risk Analysis, 35, 7, 1230-1251.

Ouyang, M, Tian, H, Wang, Z, Hong, L and Mao, Z. (2018). Critical Infrastructure Vulnerability to Spatially Localized Failures with Applications to Chinese Railway System. Risk Analysis , 39(1),

Papamichail, K.N. and S. French. (2013). 25 years of MCDA in nuclear emergency management. IMA Journal of Management Mathematics, 24, 4, 481-503.

Robertson D. Spatial Transmission Models: A Taxonomy and Framework.

Rosenhead J., Mingers J. (2001). Rational analysis for a problematic world revisited. No. 2nd. John Wiley and Sons. 
Shaw D., Franco A., Westcombe M. (2006). Problem structuring methods: new directions in a problematic world. Journal of the Operational Research Society, 57(7), 757-758.

Schneeberger K., Huttenlau M., Winter B., Steinberger T., Achleitner S., Slötter J. (2018). A Probabilistic Framework for Risk Analysis of Flood Events: A Proof-of-Concept-Study. Risk Analysis ,

Shogo Takahara, S., lijima, M., Yoneda, M. and Shimada, Y. (2018). A Probabilistic Approach to Assess External Doses to the Public Considering Spatial Variability of Radioactive Contamination and Interpopulation Differences in Behavior Pattern. Risk Analysis , 39(1),

Slovic P. (2000) The Perception of Risk. Earthscan. London, UK.

Sundell J., HaafE., Norberg T., Alén C., Karlsson M., Rosén L. (2018). Risk Mapping of Groundwater-Drawdown-Induced Land Subsidence in Heterogeneous Soils on Large Areas. Risk Analysis, 39(1),

Terti, G., Ruin , I, Gourley, J. J., Kirstetter, P., Flamig, Z., Blanchet, J., Arthur, A. and Anquetin, S. (2018). Toward Probabilistic Prediction of Flash Flood Human Impacts Risk Analysis, 39(1),

Trakas, D- (2018), Spatial Risk Analysis of Power Systems Resilience During Extreme Events. Risk Analysis, 39(1),

Vadrevu K.P., Eaturu A., Badarinath K.V.S. (2010). Fire risk evaluation using multicriteria analysis: a case study, Environmental Monitoring and Assessment, 166 (1-4): 223-239.

Von WinterfeldtD, Edwards W. (1986). Decision Analysis and Behavioral Research. New York, NY: Cambridge University Press.

Walker E., Leclerc M., Rey J-F., Beaudouin R., Soubeyrand S., Messéan A. (2018). A spatio-temporal exposure-hazard model for assessing biological risk and impact. Risk Analysis, 39(1),

Zagmutt F.J., Schoenbaum M.A., Hill A.E. (2016). The Impact of Population, Contact, and Spatial Heterogeneity on Epidemic Model Predictions. Risk Analysis, 36(5), 939-953.

Zhou Y., LiN., Wu W., Wu J., Shi P. (2014). Local Spatial and Temporal Factors Influencing Population and Societal Vulnerability to Natural Disasters. Risk Analysis, 34 (4), 614-639.

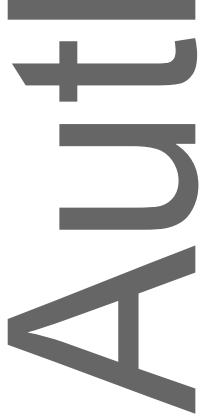

